\title{
Modeling moods in BBC programs based on emotional context
}

\author{
Petersen, Michael Kai; Butkus, Andrius
}

Published in:

Lecture Notes in Computer Science

Publication date:

2008

Document Version

Publisher's PDF, also known as Version of record

Link back to DTU Orbit

Citation (APA):

Petersen, M. K., \& Butkus, A. (2008). Modeling moods in BBC programs based on emotional context. Lecture Notes in Computer Science, 5066, 112-116.

\section{General rights}

Copyright and moral rights for the publications made accessible in the public portal are retained by the authors and/or other copyright owners and it is a condition of accessing publications that users recognise and abide by the legal requirements associated with these rights.

- Users may download and print one copy of any publication from the public portal for the purpose of private study or research.

- You may not further distribute the material or use it for any profit-making activity or commercial gain

- You may freely distribute the URL identifying the publication in the public portal

If you believe that this document breaches copyright please contact us providing details, and we will remove access to the work immediately and investigate your claim. 


\title{
Modeling Moods in BBC Programs Based on Emotional Context
}

\author{
Michael Kai Petersen and Andrius Butkus \\ Technical University of Denmark, DTU Informatics, \\ Building 321, DK.2800, Kgs.Lyngby, Denmark \\ $\{$ mkp, ab\}@imm.dtu.dk \\ http://www.imm.dtu.dk
}

\begin{abstract}
The increasing amounts of streaming and downloadable media becoming available in converged digital broadcast and next generation mobile broadband networks will require intelligent interfaces capable of personalizing the selection of content according to user preferences and moods. We propose an approach to automatically generate atmospherelike metadata from BBC synopsis descriptions, by applying LSA latent semantic analysis to define the degree of similarity between textual program descriptions and emotional tags in a semantic space.
\end{abstract}

Keywords: personalization, emotions, LSA latent semantic analysis.

\section{Introduction}

Since 2005 the broadcaster BBC has made their program listings available as XML formatted TVA TV-Anytime [1] metadata, which allows for describing media using complementary genre aspects, atmosphere as well as synopsis. We have in a related paper [2] analyzed how these metadata features may complement each other when applying more genre dimensions in parallel, and thus increase the number of relevant recommendations, by capturing similarities across the traditional divide of categories. In particular the TVA genre dimension atmosphere seemed able to identify programs that might be perceived as similar even though they belong to different genre categories. Extending this approach we propose in the present paper a method to automatically generate atmosphere-like metadata using the synopsis of TV programs. We outline in the following sections a framework for modeling emotional context using last.fm tags as markers in a semantic space, the methodology for extracting latent semantics, the retrieved results followed by a discussion of our early results based on BBC synopsis descriptions.

\section{Affective Terms}

When investigating how unstructured metadata can be used to describe media, the social music network last.fm provides an interesting case. Despite the idiosyncratic character of tags defined by hundred thousands of users, recent studies 
within music information retrieval have revealed that last.fm users often tend to agree on the emotional terms they apply to music. This correlation between social network tags and the specific music tracks they are describing, makes it possible to define high-level categories, which provide a simplified mood groundtruth reflecting the perceived emotional context of the music [3][4].

With point of departure in these findings we hypothesize that it might be possible to extract the emotional context of a TV program by projecting its synopsis into a semantic space, and use last.fm tags as affective buoys to define the textual description within emotional context. Drawing on psychological studies [5], establishing that emotional assessment can be reduced to a semantic differential spanned by the two primary dimensions of valence and arousal, we use these two axes to outline an emotional plane for a last.fm semantic tag space. The first of the these two dimensions describes how pleasant something is along an axis going from happy to sad, whereas the latter dimension captures the amount of involvement ranging from passive states like dark or soft to active aspects of excitation as reflected in tags like angry or sexy.

\section{Latent Semantics}

As a machine learning technique which resembles cognitive comprehension of text, LSA latent semantic analysis [6][7][8] extracts meaning from texts by modeling the usage patterns of words in multiple documents and represent the terms and their contexts as vectors in a high-dimensional space. To retain only the most essential features the dimensionality of the original sparse matrix is reduced to around 300 dimensions. This reduced LSA space makes it possible to compute the semantic relatedness of synopsis and affective terms as the cosine of their vectors, with values towards 1 signifying degrees of similarity between the items and low values close to zero or negative signifying a random lack of correlation. In this semantic space a synopsis text and words which express the same meaning will thus be represented as vectors that are closely aligned, even if the terms are not literally co-occurring within the same context.

\section{Results}

Taking a selection of short BBC program descriptions as input, we compute the cosine similarities between a synopsis text vector and each of the selected last.fm emotional words. An analysis of the program "News night", based on the short description: News in depth investigation and analysis of the stories behind the $\operatorname{day}(s)$ headline, triggers the tags funny and sexy which might not immediately seem a fitting description, probably caused by these emotional terms being directly correlated with the occurrence of the words stories and news within the synopsis. The atmosphere of the lifestyle program "Ready Steady Cook!" might be somewhat better reflected in the synopsis: Peter Davidson and Bill Ward challenge celebrity chefs to create mouth watering meals in minutes, which triggers the tag romantic as associated with meals. Another singular emotion can 
be retrieved from the documentary "I am boy anorexic", which based on the synopsis: Documentary following three youngsters struggling to overcome their obsessive relationship with food as they recover inside a London clinic and then return to the outside world, triggers the affective term dark. We find a broader emotional spectrum reflected in the lifestyle program "The flying gardener" described by the text: The flying gardener Chris travels around by helicopter on a mission to find Britain(s) most inspirational gardens. He helps a Devon couple create a beautiful spring woodland garden. Chris visits impressive local gardens for ideas and reveals breathtaking views of Cornwall from the air. The synopsis triggers a concentration of passive pleasant valence elements related to the words soft, mellow combined with happy. In this context also the tag cool comes out as it has a strong association to the word air contained in the synopsis, while the activation of the tag aggressive appears less explainable.
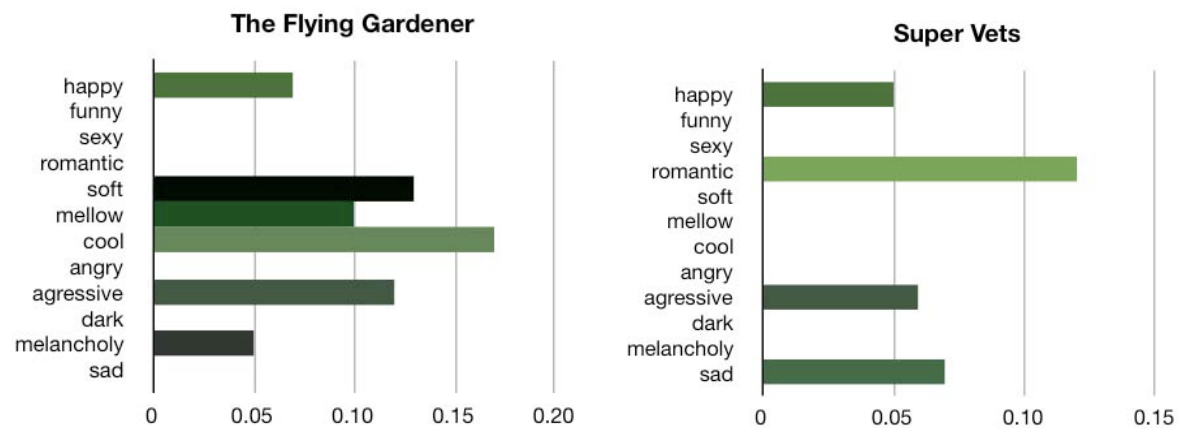

Fig. 1. LSA cosine similarity between the synopsis descriptions of "The flying gardener" and "Super Vets" against 12 frequently used last.fm affective terms

This cluster of pleasant elements is lacking in the LSA analysis of the program "Super Vets" which instead evokes a strong emotional contrast based on the text: At the Royal Vet College Louis the dog needs emergency surgery after a life threatening bleed in his chest and the vets need to find out what is causing the cat (..)fits, where both pleasant and unpleasant active terms like happy and sad stand out in combination with strong emotions reflected by the tag romantic. And as can be seen from programs like "The flying gardener" and "Super Vets" (Fig.1) the correlation between the synopsis and the chosen tags might often trigger both combinations of complementary elements as well as contrasting emotional components rather than a single monochrome feeling.

We proceeded to explore whether we could sum up a distinct pattern reflecting an emotional profile pertaining to a TV series, by accumulating the LSA values of correlation between synopsis texts and emotional tags over several episodes. For this purpose we chose the soap "East Enders" and the comedy "Two pints of lager" and analyzed descriptions of six consecutive episodes from each series.

Even when only comparing the synopsis and emotional tags over six episodes (Fig.2), it appears that the accumulated LSA correlation values in the soap "East 

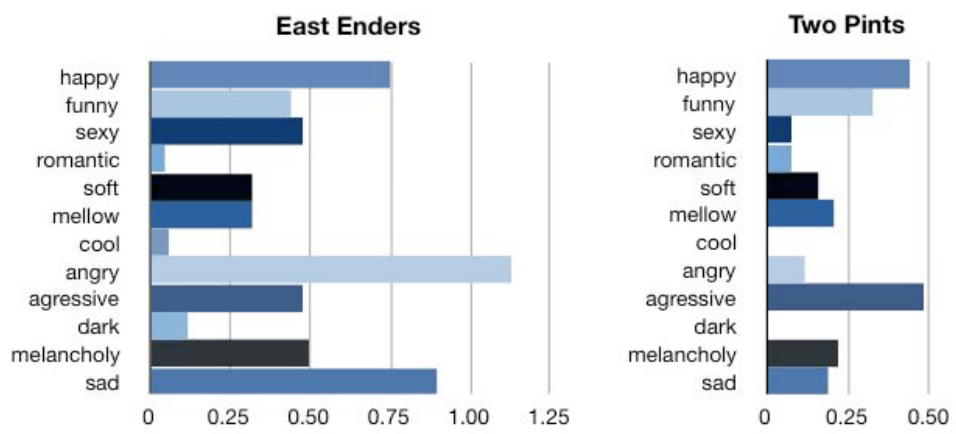

Fig. 2. LSA cosine similarity of the soap "East Enders" and the comedy "Two Pints" against 12 frequently used last.fm affective terms accumulated over six episodes

Enders" are roughly twice as high as in the comedy "Two pints of lager". The contributions of affective components in both histograms are unbalanced, but whereas the former series has a bottom-heavy emphasis on angry and sad emotions, the balance is reversed in the latter with a shift towards predominantly happy and funny elements complemented with soft and mellow aspects. These patterns can similarly be made out when considering the emotional components plotted over time for the soap and comedy respectively (Fig.3). The distribution in "East Enders" is much more dense and emotionally saturated reflecting aspects of arousal, while the character of "Two pints of lager" seems mirrored in a pronounced clustering of lighter elements of positive valence and an overall sparsity of excitation within the matrix.
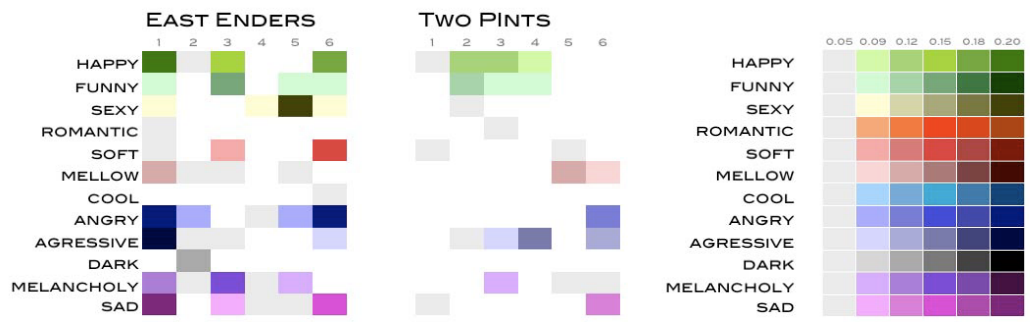

Fig. 3. LSA cosine similarity of the soap "East Enders" and the comedy "Two Pints" against 12 frequently used last.fm affective terms accumulated over six episodes

\section{Discussion}

Projecting BBC synopsis descriptions into an LSA space using last.fm tags as emotional buoys, we have demonstrated an ability to extract patterns reflecting combinations of emotional components. Analyzing the emotional components reflected in the synopsis descriptions over a sequence of episodes, we have been 
able to separate these aspects into patterns defined by the sparsity and character of the distribution. While each synopsis triggers an individual emotional response related to a specic episode, general patterns still emerge when accumulating the LSA correlation between synopsis and emotional tags over consecutive episodes, which enables us to differentiate between a comedy and a soap based on a textual description alone. We therefore propose that emotional components describing the content of media might be retrieved as latent semantics by using affective terms as sensors in a semantic space, and we suggest that LSA might be applied to extract structural patterns from synopsis descriptions as a basis for automatically generating mood-based recommendations. Though the synopsis descriptions trigger both combinations of complementary elements as well as contrasting emotional components rather than a monochrome affective response, they nevertheless pertain to distinct patterns which we speculate might be used as a basis to build emotional patterns capturing user preferences.

\section{References}

1. ETSI: TV-Anytime. Part 3. Metadata 1. Sub-part 1. Part 1 - Metadata schemas TS 102822-3-1 (2006)

2. Petersen, M., Butkus, A.: Semantic modelling using TV-Anytime genre metadata. In: Cesar, P., Chorianopoulos, K., Jensen, J.F. (eds.) EuroITV 2007. LNCS, vol. 4471, pp. 226-234. Springer, Heidelberg (2007)

3. Levy, M., Sandler, M.: A semantic space for music derived from social tags. In: Proceedings of the 8th International Conference on Music Information Retrieval, pp. 411-416. Austrian Computer Society (2007)

4. Hu, X., Bay, M., Downie, S.: Creating a simplied music mood classification groundtruth set. In: Proceedings of the 8th International Conference on Music Information Retrieval, pp. 309-310. Austrian Computer Society (2007)

5. Osgood, C.E., Suci, G.J., Tannenbaum, P.H.: The measurement of Meaning. University of Illinois Press (1957)

6. Deerwester, S., Dumais, S.T., Furnas, G.W., Landauer, T.K., Harshman, R.: Indexing by latent semantic analysis. Journal of the American Society for Information Science 41, 391-407 (1990)

7. Landauer, T.K., Dumais, S.T.: A solution to Platos problem: The latent semantic analysis theory of acquisition, induction, and representation of knowledge. Psychological Review 104, 211-240 (1997)

8. Dumais, S.T.: LSA and information retrieval: Back to basics, Handbook of latent semantic analysis, pp. 293-321. Lawrence Erlbaum, Mahwah (2007) 